Documentation et bibliothèques

DOCUMENTATION BIBLIOTHËQUES

\title{
La gestion des connaissances : concept intéressant pour les spécialistes en information documentaire ou jargon délirant ?
}

\section{France Bouthillier}

Volume 45, numéro 2, avril-juin 1999

URI : https://id.erudit.org/iderudit/1032763ar

DOI : https://doi.org/10.7202/1032763ar

Aller au sommaire du numéro

\section{Éditeur(s)}

Association pour l'avancement des sciences et des techniques de la documentation (ASTED)

\section{ISSN}

0315-2340 (imprimé)

2291-8949 (numérique)

Découvrir la revue

\section{Citer ce document}

Bouthillier, F. (1999). La gestion des connaissances : concept intéressant pour les spécialistes en information documentaire ou jargon délirant ?

Documentation et bibliothèques, 45(2), 51-51. https://doi.org/10.7202/1032763ar

Tous droits réservés (C) Association pour l'avancement des sciences et des techniques de la documentation (ASTED), 1999
Ce document est protégé par la loi sur le droit d'auteur. L'utilisation des services d'Érudit (y compris la reproduction) est assujettie à sa politique d'utilisation que vous pouvez consulter en ligne.

https://apropos.erudit.org/fr/usagers/politique-dutilisation/ 


\section{La gestion des connaissances : concept intéressant pour les spécialistes en information documentaire ou jargon délirant?}

Alors que l'expression «gestion des connaissances" frappe l'imaginaire de certains, elle en laisse plusieurs sceptiques. En effet, comment peut-on sérieusement parler de la gestion de ce qui se trouve dans le cerveau humain, alors que la gestion des ressources matérielles ou financières, sans parler de celle des ressources humaines, fait souvent l'objet d'un manque incroyable de connaissances, voire de conscience? Pourtant, le concept a donné lieu à de nombreuses publications depuis le début des années 1990. II est également associé à de nouveaux titres d'emploi surtout dans la langue de Shakespeare. On trouve, par exemple, chief knowledge officer, knowledge management professional ou intellectual asset manager. La Special Library Association y voit un terrain d'intervention privilégié pour les spécialistes en information, et elle offre régulièrement des séances de formation sur le sujet. Plusieurs grandes entreprises, et principalement des firmes de consultation, ont mis sur pied des Centres de connaissances avec gestionnaires attitrés.

Dans le monde de la gestion, l'utilisation abusive de certains termes est monnaie courante, et celle du mot gestion n'en est qu'un exemple... II n'en demeure pas moins que l'idée de répertorier, d'organiser et de rendre accessibles des connaissances à l'aide d'une infrastructure technologique dans un milieu organisationnel donné semble bel et bien faire école, mais qu'en est-il exactement?

La gestion des connaissances n'est pas un phénomène nouveau: les responsables d'entreprise se sont toujours préoccupés de la transmission des savoir-faire et des habiletés essentiels au développement de leur entreprise. Ce qui est nouveau, c'est la volonté d'en faire un processus formel. Après la vague de rationalisations en série, beaucoup d'entreprises ont remercié des centaines de personnes qui sont parties sans avoir transmis ce qu'elles savaient. Ceci a engendré bien souvent des situations chaotiques, et la performance en a pris un coup. Par ailleurs, des outils technologiques récemment développés permettent de codifier, de stocker, d'organiser et de rendre disponible l'information, peu importe sa localisation et son format, à des groupes d'employés qui peuvent se l'approprier en temps voulu comme jamais auparavant. D'où l'intérêt grandissant pour la gestion du savoir particulièrement dans une économie où le capital intellectuel est clairement devenu une source d'avantage concurrentiel. Or, certaines formes de connaissances ne peuvent être codifiées. Selon Thomas H. Davenport et Laurence Prusak (Working Knowledge: How Organizations Manage What They Know, Boston, Mass: Harvard Business School Press, 1998), plus les connaisances sont complexes et intégrées dans l'expérience individuelle, donc tacites, moins il est possible de les codifier et de les rendre disponibles dans un document ou une base de données. II est donc impossible, et il serait futile, de vouloir gérer toutes les connaissances utilisées dans un environnement de travail. La gestion des connaissances doit permettre d'atteindre des objectifs précis, sinon elle risque de devenir une stratégie vide d'intérêt.

Aussi abusif qu'il puisse paraître, le concept mérite d'être examiné sérieusement par les spécialistes en information, car il est intimement lié à la gestion stratégique de l'information et à la gestion de systèmes d'information. Toutefois, il serait naif de penser qu'il sera facile pour ces derniers de prendre leur place dans ce domaine déjà dominé par des experts en recettes technologiques, qui ne sont pas nécessairement préoccupés par les besoins organisationnels des entreprises.

Dans les textes qui suivent, Denis Levasseur nous donne un exemple intéressant de projet de gestion des connaissances. II explique l'implantation d'une infrastructure documentaire pour des groupes de travail ayant des besoins particuliers, qui illustre bien l'utilisation des intranets dans les organisations. Cette technologie permet de créer un espace virtuel, lequel remplace de plus en plus la bibliothèque spécialisée. L'auteur fait bien ressortir à quel point le rôle du conseiller en information s'en trouve modifié.

On l'a maintes fois dit, les nouvelles technologies de l'information transforment le rapport que l'on entretient avec l'information et le document. Le texte de Suzanne Bertrand-Gastaldy et de Paul Marchand nous offre un exemple de ce que l'on peut découvrir dans un texte littéraire en utilisant un logiciel d'analyse de textes par ordinateur. Selon les auteurs, de tels logiciels ouvrent également de nouvelles avenues pour les spécialistes de la documentation intéressés à développer de nouveaux types de services documentaires reliés à l'analyse de textes.

Dans le présent numéro, nous avons également le privilège de publier un texte d'un collaborateur parisien, Pierre-Alain Tilliette, conservateur à la Bibliothèque administrative de la Ville de Paris. II nous présente une description de la bibliothèque, son rôle ainsi qu'une partie de son histoire. Cette chronique nous permet de réfléchir sur l'importance des institutions, relativement aux technologies de l'information, dans la mise en place d'un réseau d'accès aux connaissances.

\section{France Bouthillier}

